\title{
Childhood sarcoidosis: long-term follow-up
}

\author{
N. Milman* and A.L. Hoffmann ${ }^{\#}$
}

ABSTRACT: The aim of the present study was to describe clinical features and long-term survival in childhood sarcoidosis.

In total, 46 ethnic Caucasian Danish children (aged $<16$ yrs, 24 males) with sarcoidosis were identified in 1979-1994. In 33 (72\%) children, diagnosis was verified by histology and, in the remaining 13 , by clinical and radiological findings. In total, 37 subjects had a follow-up examination. Median (range) age at onset of disease was 14 (0.7-15.8) yrs and median (range) clinical follow-up was 15 (3-23) yrs after onset of disease. The median (range) age at clinical follow-up was 28 (17-30) yrs.

At follow-up: 36 (78\%) children recovered completely; 30 (65\%) showed complete clinical regression at a median (range) $0.7(0.6-5.9)$ yrs after onset of disease; two (4\%) recovered with organ damage (unilateral loss of vision, abnormal chest radiograph); five (11\%) still had chronic active disease with multiorgan involvement and impaired lung function; and three (7\%) were deceased, due to central nervous system sarcoidosis and acute myeloid leukaemia probably caused by cytostatics.

In Danish children, sarcoidosis had a favourable prognosis; the majority recovered $<\mathbf{6}$ yrs after onset of disease. Some developed chronic active disease and impairment of pulmonary function, demanding continuous medical treatment. Prognosis was not related to the age at onset of disease. Erythema nodosum was associated with a good prognosis, and central nervous system involvement with a poor prognosis.

\section{KEYWORDS: Children, follow-up, granulomatosis, sarcoidosis}

arcoidosis is a disease of unknown aetiology, characterised by the formation of nonnecrotising epithelioid cell granulomas with multiorgan involvement [1]. The overall incidence of sarcoidosis in Denmark is 7.2/ 100,000 person-yrs, with the peak incidence occurring at 230 yrs of age [2]. Among Danish children, the incidence rises from $0.06 / 100,000$ person-yrs at age $\leqslant 4$ yrs to $1.02 / 100,000$ person-yrs at age 14-15 yrs with an overall incidence of 0.29 / 100,000 person-yrs [2, 3]. The incidence declines from the Western to the Eastern part of Denmark both in adults [2] and in children [3]. The regional variations in incidence rates are probably due to regional differences in diagnostic awareness, although environmental and genetic differences might also be involved [2].

The natural history of sarcoidosis has been studied most extensively in adults [2, 4-6]. In contrast, the natural history and prognosis of sarcoidosis has been scarcely investigated in children [3]. Consequently, the objective of the present study was to describe the long-term course and prognosis of childhood sarcoidosis in Denmark, especially with respect to clinical outcome and vital prognosis.

\section{PATIENTS AND METHODS}

The study was approved by the ethics committee in the region of Copenhagen. In Denmark, patients discharged from any hospital are registered according to their diagnoses in a Nationwide Patient Registry, established in 1979 and hosted by the National Board of Health. Patients with a diagnosis of sarcoidosis in the period 1979-1994 were drawn from this National Patient Registry [3]. In total, 5,536 patients were drawn from the Registry; 81 patients were aged $<16$ yrs. By reassessment of the patient records and registry information, the diagnosis of sarcoidosis could be reconfirmed in $49(61 \%)$ patients, i.e. in one more patient than in the initial series [3].

From the present series of 49 patients, three patients were excluded: a pair of monozygotic twins, who later proved to have Blau syndrome [7] and an ethnic Lebanese young male who had returned to his country and was unavailable for follow-up. The final series thus comprised 24 males and 22 females (male/female ratio 1:1), all ethnic Caucasian Danes. In 33 (72\%) patients, the diagnosis was verified by examination of tissue biopsy specimens showing sarcoid granulomas. In 13 patients, characteristic chest radiograph
AFFILIATIONS

*The Heart Centre, Division of Lung Transplantation, Rigshospitalet, University of Copenhagen, Copenhagen, and \# Dept of Paediatrics, Næstved Hospital, Næstved, Denmark.

CORRESPONDENCE

N. Milman

Lindevangen 87B

DK-2830 Virum

Denmark

Fax: 4535452648

E-mail: nils.mil@dadlnet.dk

Received:

January 312007

Accepted after revision:

November 122007

SUPPORT STATEMENT

This work was supported by Dronning Louises Børnehospitals

Forskningsfond

STATEMENT OF INTEREST

None declared. 
findings (hilar adenopathy) associated with typical clinical features and/or laboratory findings (erythema nodosum (EN), uveitis, peripheral lymphadenopathy and elevated serum angiotensin-converting enzyme (SACE)) substantiated the diagnosis [3].

The subjects were invited by letter to a clinical follow-up examination in November, 1999. In total, 37 subjects responded and had a clinical examination, which is herein referred to as "clinical follow-up". At closure of the study in September, 2006, vital status on all 46 subjects was checked in the National Census Registry, which is herein referred to as "Registry follow-up".

Clinical follow-up comprised a history, clinical examination, blood samples for biochemical analyses performed by standard laboratory methods and chest radiograph. Chest radiograph findings were scored in a blinded fashion by the present authors. Blood pressure was measured with a mercury manometer. Pulmonary function tests, including diffusing capacity of the lung for carbon monoxide (DL,CO), were performed using a body plethysmograph (Medical Graphics, St Paul, MN, USA).

\section{RESULTS}

\section{Duration of follow-up}

Table 1 shows the duration of follow-up and the age at followup. Median (range) age at onset of symptoms was 14.0 (0.7$15.8)$ yrs; one patient was aged $0-4$ yrs, 10 patients were 5 11 yrs and 35 patients were $12-15$ yrs. The age at the diagnosis of sarcoidosis was slightly higher with a median (range) of 14.5 (3.8-16.3) yrs.

Two (4\%) patients reported sarcoidosis in the family, one mother and one father, who both had recovered from the disease.

\section{Clinical features at onset of disease}

Clinical, laboratory and radiological features at the onset of disease have been described previously [3]. General malaise, weight loss, fever, respiratory symptoms, lymphadenopathy, skin manifestations and ocular and central nervous system (CNS) symptoms were frequent at onset of disease, whereas arthritis was infrequent [3]. The initial cardinal symptom/ clinical finding at the onset of disease for which the patients contacted the healthcare system is shown in table 2 .

\section{Treatment}

The children were followed and treated in the regional departments of paediatrics all over Denmark. There were no approved common guidelines for treatment, which was at the discretion of the local doctors, and it is beyond the scope of the present study to analyse the effect of therapy. Treatment was preferably given to patients with clinically overt pulmonary involvement, with extrathoracic organ involvement (e.g. CNS involvement) and with hypercalcaemia; 23 (51\%) out of 45 (data were not available for one patient) of the children were treated with prednisolone for median (range) 1.3 (0.3-23) yrs. Two children were additionally treated with methotrexate (patient 1) or azathioprine (patient 2).

\section{Outcome at follow-up}

In $30(65 \%)$ subjects, there was complete clinical regression at median (range) $0.7(0.6-5.9)$ yrs after onset of symptoms. At follow-up, $36(78 \%)$ of the subjects had recovered completely and appeared fit without health-related problems from sarcoid disease (table 3).

Five patients had chronic active sarcoidosis as follows. 1) A 4.3yr-old (at onset) female (patient 3) had permanent impairment of lung function and active sarcoid colitis/proctitis at followup 18 yrs later. 2) A 0.7-yr-old (at onset) male (patient 1) had chronic active pulmonary sarcoidosis with impaired lung function at follow-up 24 yrs later. 3) A 15.3-yr-old (at onset) male (patient 4) with pulmonary sarcoidosis stage II and neurosarcoidosis, with impaired visual field, hypophyseal insufficiency and diabetes insipidus, was still on treatment at follow-up 21 yrs later. 4) A 14.5-yr-old (at onset) female (patient 5) with iridocyclitis, stage I pulmonary sarcoidosis and neurosarcoidosis with diabetes insipidus still had active stage I pulmonary sarcoidosis with normal lung function tests and diabetes insipidus at follow-up 10 yrs later. 5) A 13.3-yr-old (at onset) female (patient 6) with hypercalcaemia, nephrocalcinosis and stage I pulmonary sarcoidosis still had active stage III pulmonary sarcoidosis with impaired lung function at followup 23 yrs later.

\section{Anthropometrics}

Anthropometrics were available in 31 subjects (14 males, 17 females). The mean (range) height in males was 1.80 (1.661.94) $\mathrm{m}$. This height was below the age-adjusted sex-specific mean height in Danes $(1.82 \mathrm{~m})$ in nine $(64 \%)$ males and two had a height below the 5 th percentile $(1.70 \mathrm{~m})$. Of these, one had a benign course of disease without steroids and was fit and well and the other had chronic active sarcoidosis (patient 7) and had been on steroids for 23 yrs. The mean (range) height in females was $1.67(1.57-1.78) \mathrm{m}$. This height was below the age-adjusted sex-specific mean height in Danes $(1.69 \mathrm{~m})$ in 11 $(65 \%)$ females. Of these, one (patient 3$)$ had a height below the 5 th percentile $(1.59 \mathrm{~m})$, had chronic active sarcoidosis and had been on steroids for 6 yrs.

TABLE 1 Follow-up of children with sarcoidosis diagnosed in 1979-1994 in Denmark

Clinical follow-up 1999 37

Subjects $\mathrm{n}$

Follow-up after onset of sarcoidosis yrs

Age at follow-up yrs

Registry follow-up 2006

46

$23(4-30)$

$29(18-42)$

Data are presented as median (range), unless otherwise stated. 


\begin{tabular}{ll}
\hline TABLE 2 & $\begin{array}{l}\text { Initial cardinal features at onset of disease in } 46 \\
\text { patients with childhood sarcoidosis }\end{array}$ \\
Initial symptom & Subjects $\mathbf{n}$ \\
\hline Erythema nodosum & 10 \\
Iridocyclitis & 10 \\
Peripheral lymphadenopathy & 7 \\
Skin sarcoidosis & 3 \\
Scar sarcoidosis & 2 \\
Rhinitis and/or sinusitis & 2 \\
Cough and/or exertional dyspnoea & 2 \\
Fever & 2 \\
Hypercalcaemic symptoms & 2 \\
Parotid swelling & 1 \\
Facial palsy & 1 \\
Abdominal pain & 1 \\
Diarrhoea & 1 \\
No symptoms, by incidence & 2 \\
Total & 46 \\
\hline
\end{tabular}

Mean body mass index (BMI) was $24.3 \mathrm{~kg} \cdot \mathrm{m}^{-2}$. One $(3 \%)$ female patient was underweight (BMI $\left.<18.5 \mathrm{~kg} \cdot \mathrm{m}^{-2}\right)$; $\operatorname{six}(19 \%)$, two males and four females were moderately overweight (BMI $\left.25.0-29.9 \mathrm{~kg} \cdot \mathrm{m}^{-2}\right)$, and three $(10 \%)$, two males and one female were obese $\left(B M I \geqslant 30 \mathrm{~kg} \cdot \mathrm{m}^{-2}\right)$.

\section{Organ involvement and course of disease}

Table 4 shows the pattern of organ involvement at onset of disease and at follow-up. All children presenting with EN were fit and healthy at follow-up.

Three children had sarcoid skin lesions and one male (patient 1) with onset of sarcoidosis at 0.7 yrs of age still had chronic active pulmonary disease.

In total, 13 children presented with uveitis/iridocyclitis. One had permanent unilateral loss of vision, but no activity in ocular disease at follow-up and one still had chronic active sarcoidosis, but no activity in ocular disease at follow-up.

The majority of children presented with mediastinal/hilar lymphadenopathy: 11 children presented with peripheral lymphadenopathy, predominantly on the neck. Of these, nine children recovered, one had sequela with unilateral loss of vision (table 4) and one patient was deceased.

Of the two patients presenting with abdominal pain, a 15.6-yrold male had had a laparotomy. Mesenteric lymph nodes were enlarged and examination of appendix vermiformis showed noncaseating epithelioid cell granulomas [3]. This patient recovered completely. The second patient, a 4.3-yr-old female, had sarcoid involvement of colon and rectum and at follow-up she still had chronic active sarcoidosis involving the colon and lungs (stage IV).

Two children had peripheral facial palsy and iridocyclitis, of which, one recovered completely, and the other died of acute myeloid leukaemia. Three children aged 11.2-15.3 yrs at onset of disease had CNS involvement and one, in addition, had

\section{TABLE 3 Outcome after childhood sarcoidosis in 46 patients diagnosed in 1979-1994}

Subjects

$\begin{array}{lc}\text { Fully recovered } & 36(78) \\ \text { Recovered with sequelae } & 2(4) \\ \text { Chronic active disease with organ damage } & 5(11) \\ \text { Deaths } & 3(7) \\ \quad \text { From central nervous system sarcoidosis } & 2 \\ \text { From acute myeloid leukaemia associated with } & 1 \\ \quad \text { treatment } & \end{array}$

Data are presented as $n$ or $n(\%)$. Two patients recovered with sequelae: 1) a 14.6-yr-old (at onset) male recovered with unilateral loss of vision due to sarcoid uveitis, but without activity in ocular disease at follow-up; and 2) a 12.2-yr-old (at onset) male had persistent radiographic stage II at follow-up, but normal pulmonary function tests.

facial palsy. At follow-up, one patient still had chronic active sarcoidosis and two were deceased.

None of the patients presented with clinically significant sarcoid-associated arthritis.

\section{Laboratory analyses at follow-up}

Biochemical blood analyses at follow-up were available in 35 subjects (17 males, 18 females; table 5 ). The majority of subjects had values within the normal reference interval. All subjects had normal blood haemoglobin, blood erythrocyte counts and erythrocyte indices (mean corpuscular volume (MCV) and mean corpuscular haemoglobin concentration), except in one female with low MCV due to iron deficiency. Blood leukocyte counts, differential counts and platelets were normal, except in one male with slight lymphopaenia due to steroid treated chronic active sarcoidosis. Plasma albumin, sodium, potassium, bicarbonate, urea, creatinine and erythrocyte sedimentation rate (ESR) were normal, except in two females with slightly elevated ESR $\left(\leqslant 25 \mathrm{~mm} \cdot \mathrm{h}^{-1}\right)$. Of these, one had nonclassified rheumatic disease with arthralgias and the other was healthy.

Serum soluble interleukin-2 receptor (S-sIL-2R) was measured in 28 subjects, in 24 who had recovered and in four with chronic active disease. All subjects who had recovered had normal S-sIL-2R levels, whereas all four subjects with active disease had elevated levels (table 5).

Arterial blood pressure was normal, with a median of 119 / $80 \mathrm{mmHg}$ in 29 out of 30 subjects. One male was diagnosed with arterial hypertension $(160 / 123 \mathrm{mmHg})$ at follow-up.

\section{Chest radiology}

Table 6 shows the chest radiograph findings. At follow-up, chest radiographs were available in 39 subjects. In the seven subjects where chest radiographs were not taken at follow-up, radiology at diagnosis showed stage 0 in one patient, stage I in four patients and stage II in two patients. The majority of the 39 subjects $(\mathrm{n}=31)$ had overall "normal" chest radiographs; however, $16(52 \%)$ of the 31 subjects had sequelae after mediastinal/hilar lymphadenopathy in the form of mediastinal 
TABLE 4 Organ involvement in childhood sarcoidosis at onset of disease and at follow-up

\begin{tabular}{|c|c|c|c|}
\hline & \multirow[t]{2}{*}{ Onset of disease } & \multicolumn{2}{|r|}{ Follow-up } \\
\hline & & Recovered & Disease if not recovered \\
\hline Upper respiratory tract & $6(13)$ & 6 & \\
\hline \multicolumn{4}{|l|}{ Lower respiratory tract } \\
\hline Abnormal chest radiograph & $42(91)$ & $33^{\#}$ & 1 sequela (stage II) \\
\hline \multicolumn{4}{|l|}{ Skin manifestations } \\
\hline Erythema nodosum & $10(22)$ & 10 & \\
\hline Scar sarcoidosis & $2(4)$ & 2 & \\
\hline Sarcoid skin lesions & $3(7)$ & 2 & 1 chronic active sarcoidosis \\
\hline \multicolumn{4}{|l|}{ Lymph nodes } \\
\hline Peripheral lymphadenopathy & $11(24)$ & 9 & 1 sequela (unilateral loss of vision) \\
\hline Mesenteric lymphadenopathy & $1(2)$ & 1 & \\
\hline \multirow[t]{3}{*}{ Hypercalcaemia } & $13(28)$ & 10 & 1 sequela (unilateral loss of vision) \\
\hline & & & 1 chronic active sarcoidosis \\
\hline & & & 1 deceased (acute leukaemia) \\
\hline \multicolumn{4}{|l|}{ Ocular involvement } \\
\hline \multirow[t]{3}{*}{ Iridocyclitis/uveitis } & $13(28)$ & 9 & 1 sequela (unilateral loss of vision) \\
\hline & & & 1 chronic active sarcoidosis \\
\hline & & & 2 deceased \\
\hline Neurosarcoidosis & $5(11)$ & & \\
\hline Peripheral neuropathy & $2(4)$ & 1 & 1 deceased (acute leukaemia) \\
\hline \multirow[t]{2}{*}{ Central nervous system } & $3(7)$ & & 1 chronic active sarcoidosis \\
\hline & & & 2 deceased (neurosarcoidosis) \\
\hline
\end{tabular}

Data are presented as $\mathrm{n}(\%)$ or $\mathrm{n}$. *: two subjects with initial stage I made a complete clinical recovery with normal lung function, but had no chest radiograph at follow-up

$\because$ chronic active sarcoidosis indicates that the disease is in a chronic active state and may or may not involve the organ listed.

TABLE 5 Results of laboratory analyses at follow-up of patients who had sarcoidosis in childhood

\begin{tabular}{|c|c|c|c|c|}
\hline Variable & Subjects $n$ & Median (range) & Reference interval & Abnormal $n$ \\
\hline Plasma total calcium $\mathrm{mmol} \cdot \mathrm{L}^{-1}$ & 35 & $2.52(2.21-2.73)$ & $2.20-2.60$ & $3^{\#}$ \\
\hline Plasma ionised calcium $\mathrm{mmol} \cdot \mathrm{L}^{-1}$ & 32 & $1.25(1.19-1.41)$ & $1.17-1.34$ & $1^{\bullet}$ \\
\hline Plasma IgG $\mu \mathrm{mol} \cdot \mathrm{L}^{-1}$ & 33 & $73(43-95)$ & $41-99$ & \\
\hline Plasma IgA $\mu \mathrm{mol} \cdot \mathrm{L}^{-1}$ & 33 & $12.4(4.0-23.1)$ & $4.4-22.8$ & $2^{+}$ \\
\hline Plasma IgM $\mu \mathrm{mol} \cdot \mathrm{L}^{-1}$ & 33 & $0.93(0.29-1.92)$ & $0.41-2.19$ & $2^{\S}$ \\
\hline Serum soluble interleukin-2 receptor $\mathrm{U} \cdot \mathrm{L}^{-1}$ & 28 & $469(317-1660)$ & $223-710$ & $4^{\mu}$ \\
\hline
\end{tabular}

Ig: immunoglobulin; SACE: serum angiotensin-converting enzyme. " : one female with hypercalcaemia at diagnosis also had high ionised calcium at follow-up and was otherwise healthy, one female and one male without hypercalcaemia at diagnosis had normal ionised calcium at follow-up and were healthy; ": one female with

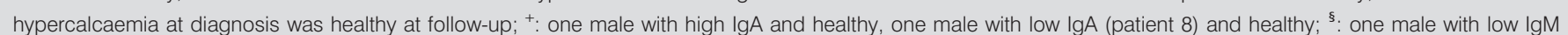
(patient 8) and healthy, one male with low IgM (patient 1) with steroid-treated chronic active pulmonary sarcoidosis; ${ }^{f}$ : one female with sarcoid diabetes insipidus and no allergy (patient 5), one female with chronic active stage III pulmonary sarcoidosis and no allergy (patient 6); \#\#: one female with chronic active stage III pulmonary sarcoidosis (patient 6); " " one female with serum soluble interleukin-2 receptor (S-sIL-2R) $876 \mathrm{U} \cdot \mathrm{L}^{-1}$, SACE $113 \mathrm{U} \cdot \mathrm{L}^{-1}$, chronic active stage I pulmonary sarcoidosis and diabetes insipidus and not on steroids (patient 5), one female with S-SIL-2R $1112 \mathrm{U} \cdot \mathrm{L}^{-1}$, SACE $32 \mathrm{U} \cdot \mathrm{L}^{-1}$, chronic active stage IV pulmonary sarcoidosis and intestinal sarcoidosis and was not on steroids, one female (patient 6) with S-sIL-2R $1280 \mathrm{U} \cdot \mathrm{L}^{-1}$, SACE $130 \mathrm{U} \cdot \mathrm{L}^{-1}$ with chronic active stage III pulmonary sarcoidosis and nephrocalcinosis and not on steroids, one male (patient 1) with S-SIL-2R $1680 \mathrm{U} \cdot \mathrm{L}^{-1}$, SACE $85 \mathrm{U} \cdot \mathrm{L}^{-1}$ with chronic active stage I pulmonary sarcoidosis and on steroids. 
scar formation or mediastinal fibrosis. Median time to regression of chest radiograph abnormalities in the 31 children was 2.2 $(0.6-5.9)$ yrs and stages II-IV were seen in four $(10 \%)$ of the 39 patients. Mediastinal/hilar calcifications were noted in three $(8 \%)$ of the subjects.

Changes in chest radiograph findings are shown in figure 1; three out of three patients with stage 0,20 out of 24 patients with stage I, seven out of 10 patients with stage II and one out of two patients with stage III at onset of disease had a normal chest radiograph at follow-up.

\section{Lung function}

Table 7 shows the results of pulmonary function tests at follow-up in 33 subjects. In total, 30 subjects had normal lung function, whereas three subjects with chronic active pulmonary sarcoidosis (patients 1, 3 and 6) had impaired lung function.

\section{Sarcoidosis in the young child}

In one male (patient 1 ) the disease started at 8 months of age with fever and facial erythema. A skin biopsy was interpreted as panniculitis and vasculitis, but later revision disclosed granulomas. A repeat skin biopsy at 16 months of age showed epithelioid cell granulomas. Subsequently, the child developed iridocyclitis, splenomegaly, hypercalcaemia and elevated SACE. Chest radiograph showed hilar lymphadenopathy (stage I). The patient had been on life-long treatment with prednisolone. At one point, he was treated with methotrexate, which was discontinued due to hypo- $\gamma$-globulinaemia. At 25 yrs of age, the patient still presented with chronic active pulmonary sarcoidosis, chest radiograph stage I and pulmonary function tests showing normal spirometry values but decreased $D$ L,CO $44 \%$ of predicted value and elevated S-sIL-2R. DNA sequencing showed no Blau syndrome-associated mutations in the exon 4 of the NOD2 (CARD15) gene.

\section{Malignancy}

One male with onset of sarcoidosis at 14.6 yrs of age, who had recovered from the disease, was successfully operated for seminoma of the testis at the age of 19 yrs. One male with onset

\begin{tabular}{lcc}
\hline TABLE 6 & $\begin{array}{l}\text { Chest radiograph stage at diagnosis and at } \\
\text { follow-up of patients with sarcoidosis in } \\
\text { childhood }\end{array}$ \\
$\begin{array}{l}\text { Chest radiograph } \\
\text { stage }^{\#}\end{array}$ & Diagnosis & Follow-up \\
\hline 0 & $4(9)$ & $31(80)$ \\
I & $28(61)$ & $4(10)$ \\
II & $12(26)$ & $2(5)$ \\
III & $2(4)$ & $1(3)$ \\
IV & 0 & $1(3)$ \\
Total $\mathbf{n}$ & 46 & 39 \\
\hline
\end{tabular}

Data are presented as $n$ (\%). Stage 0 can be described as normal; stage I as presenting mediastinal/hilar lymphadenopathy; stage II as mediastinal/hilar lymphadenopathy and parenchymal infiltrates; stage III as parenchymal infiltrates; stage IV as pulmonary fibrosis. of sarcoidosis at $11.2 \mathrm{yrs}$, who had recovered with organ impairment, died aged 21 yrs from acute myeloid leukaemia, probably induced by former treatment with a cytostatic.

\section{Mortality}

The three deaths in the present study were related to the sarcoid disease as follows. 1) A 15.3-yr-old (at onset) male with pulmonary sarcoidosis stage II, chronic iridocyclitis and CNS sarcoidosis verified by magnetic resonance imaging, died aged 19 yrs in status epilepticus. An autopsy was not performed. 2) A 14.9-yr-old (at onset) female with pulmonary sarcoidosis stage II and CNS sarcoidosis with facial nerve palsy and obstructive hydrocephalus was treated with an intracerebral shunt and died aged 32 yrs from cerebral infarctions. An autopsy was not performed. 3) A 11.2-yr-old (at onset) male (patient 2) with iridocyclitis, facial palsy, peripheral lymphadenopathy, hypercalcaemia, pulmonary sarcoidosis stage II and active sarcoid disease for $>6$ yrs causing permanent impairment of lung function was treated with azathioprine for 18 months in addition to prednisolone. He died aged 21 yrs from acute myeloid leukaemia, probably induced by the cytostatic therapy. An autopsy showed haemorrhagic diathesis and fungal sepsis. The mediastinal and retroperitoneal lymph nodes were adherent in large conglomerates; histological examination showed hyalinisation and fibrosis with no active sarcoid granulomas. The other organs, including the brain, contained no granulomas.

\section{DISCUSSION}

The incidence, clinical picture and prognosis of sarcoidosis display marked racial differences and the majority of studies on children have presented patient series containing subjects of different ethnic origins [8, 9, 10-14]. The present study describes the initial clinical presentation and outcome of sarcoidosis in a racially uniform consecutive series of Danish children.

The four most frequent initial presenting symptoms were EN in $22 \%$, iridocyclitis in $22 \%$, peripheral lymphadenopathy in $15 \%$ and cutaneous sarcoidosis in $7 \%$ of the patients. Arthritis was not a prominent feature in the series, even in children with EN. This observation is in contrast to previous series of childhood sarcoidosis, which emphasised that arthritis is a common symptom in small children with the disease [15, 16]. However, those patients were racially heterogenous and were not examined for mutations of the NOD2 gene. The present authors' original series [3] comprised a pair of monozygotic twins having early onset disease with arthritis/periarthritis. They were subsequently diagnosed by genetic analysis as having Blau syndrome [7]. It is possible that many children previously classified as "early onset sarcoidosis" may in fact have Blau syndrome if properly investigated for NOD2 mutations. In the present series, one child had early onset sarcoidosis at 8 months of age. He developed skin sarcoidosis, iridocyclitis and had intrathoracic involvement, but no joint symptoms and no Blau associated mutations. He was extensively investigated for autoimmune disease/vasculitis with negative results. The present authors concluded that the patient had "true" early onset sarcoidosis, which appears to be extremely rare in children aged $<1 \mathrm{yr}$. 


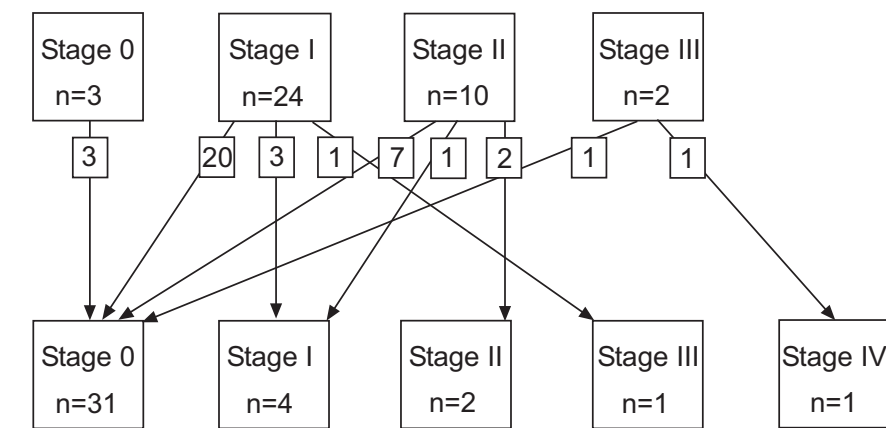

FIGURE 1. Changes in chest radiograph findings from diagnosis of disease to follow-up in 39 patients with sarcoidosis in childhood.

In general, the prognosis of childhood sarcoidosis in the present group of patients was quite good. In total, $80 \%$ of the subjects recovered completely without functional impairment. However, $\sim 20 \%$ suffered from chronic active disease with organ damage or had succumbed from sarcoidosis per se or from complications related to treatment of the disease. Thus, the overall prognosis appears to be similar to the prognosis in adults from the nordic countries [6].

EN appeared to be an important prognostic marker. Children presenting with EN had a favourable prognosis compared with children without EN. Similarly, children with scar sarcoidosis and peripheral lymphadenopathy had a good prognosis. Hypercalcaemia, iridocyclitis and CNS involvement were associated with a less favourable prognosis.

The majority of subjects had anthropometrics that corresponded to the normal population. Three subjects had a height below the 5th percentile for age. Of these, one had not been treated with steroids and had recovered completely from sarcoidosis, whereas two had been on steroids for years due to chronic active disease. In the entire group of patients, one female was slightly underweight, had a complete recovery from sarcoidosis and was otherwise fit and healthy. In contrast, overweight was a common finding: 19\% were moderately overweight and $10 \%$ were obese. These figures are comparable to the general population.

Newly discovered arterial hypertension was found in one male at the follow-up examination. He had a normal BMI of $21 \mathrm{~kg} \cdot \mathrm{m}^{-2}$ and had smoked for 15 pack-yrs.

Hypercalcaemia was found in $28 \%$ of the children at onset of disease, four of which had symptomatic hypercalcaemia [17]. In another study [8], hypercalcaemia and hypercalciuria have been reported in $5-35 \%$ of the children. Hypercalciuria may occur even in the presence of normocalcaemia and may contribute to nephrolithiasis and nephrocalcinosis [16, 18]. Four of the children in the present study had temporarily impaired renal function due to hypercalcaemia and one had nephrocalcinosis at the initial presentation. Renal dysfunction is most often due to hypercalcaemia, but may occasionally be elicited by granulomatous interstitial nephritis [16]. In general, laboratory values at follow-up were within the normal reference intervals. Three subjects had slightly elevated plasma (P-) total calcium at follow-up, but only one of these had elevated P-ionised calcium as well. The disease activity marker

\begin{tabular}{|c|c|c|c|}
\hline $\begin{array}{ll}\text { TABLE } 7 & \text { Pul } \\
& \text { chil }\end{array}$ & $\begin{array}{l}\text { ary function tes } \\
\text { d sarcoidosis }\end{array}$ & ts at follow-up & \\
\hline Lung function test & $\begin{array}{c}\text { Abnormal value } \\
\%\end{array}$ & $\begin{array}{c}\text { Median (range) } \\
\% \text { pred }\end{array}$ & $\begin{array}{c}\text { Abnormal } \\
n / N\end{array}$ \\
\hline vc & $<80$ & $101(45-147)$ & $1 / 33^{\#}$ \\
\hline FVC & $<80$ & $103(50-154)$ & $2 / 33^{\circ}$ \\
\hline $\mathrm{FEV}_{1}$ & $<80$ & $104(47-139)$ & $2 / 33^{\circ}$ \\
\hline FEV $_{1} /$ FVC & $<80$ & $98(81-109)$ & $0 / 33$ \\
\hline TLC & $<80$ & $102(67-130)$ & $1 / 31^{+}$ \\
\hline $\mathrm{RV} / \mathrm{TLC}$ & $>125$ & $91(61-214)$ & $2 / 31^{\S}$ \\
\hline$D L, C O$ & $<80$ & $99(44-134)$ & $2 / 31^{f}$ \\
\hline DL,CO/VA & $<80$ & $98(58-128)$ & $2 / 30^{f}$ \\
\hline
\end{tabular}

$\%$ pred: \% predicted; $\mathrm{n}$ : number of subjects who presented abnorma pulmonary function tests; $\mathrm{N}$ : total number of subjects tested; VC: vital capacity; FVC: forced vital capacity; FEV1: forced expiratory volume in one second; TLC: total lung capacity; RV: residual volume; $D \mathrm{~L}, \mathrm{CO}$ : diffusing capacity of the lung for carbon monoxide; VA: alveolar volume. \#: one female (patient 3) had VC $45 \%$ pred with chronic active pulmonary sarcoidosis; ${ }^{\bullet}$ : two females (patients 3 and 6) had FVC 50 and $75 \%$, and FEV 17 and $58 \%$, respectively, both with chronic active pulmonary sarcoidosis; ${ }^{+}$: one female (patient 3) had TLC $67 \%$ with chronic active pulmonary sarcoidosis; ${ }^{\text {§: }}$ two females (patients 3 and 6) had RV/ TLC 214 and 147\%, respectively, both with chronic active pulmonary sarcoidosis; ${ }^{f}$ : one female (patient 6) had DL,CO 54\% and DL,CONA 76\%, and one male (patient 1) had $D L, C O 44 \%$ and DL,CONA $58 \%$, both with chronic active pulmonary sarcoidosis

SACE was elevated in one female with chronic active sarcoidosis. S-sIL-2R levels were normal in subjects who had recovered, while patients with chronic active disease had elevated levels. Consequently, the present authors assume that S-sIL-2R is a clinically useful marker of sarcoid disease activity [19].

Involvement of the peripheral nervous system and CNS is infrequent in adult Danish sarcoidosis patients affecting $\sim 1-$ $2 \%$ [5]. However, neurological involvement appeared to be more common in children ( $4 \%$ had facial palsy, $7 \%$ had CNS involvement) and was associated with a poor prognosis.

As in adults [5], the most common finding in childhood sarcoidosis is an abnormal chest radiograph $[8,12,13]$. In the present series, $>90 \%$ had abnormal chest radiograph at onset of disease, stage I in two thirds and stages II-III in one third of the subjects. At follow-up: $80 \%$ had a normal chest radiograph, although some of these subjects had slight mediastinal scarring, some with calcification of the mediastinal lymph nodes; $10 \%$ had chronic persistent stage I; and $10 \%$ had stages II-IV. This implies that $21 \%$ of the subjects presented with an abnormal chest radiograph several years after onset of the disease. However, only $9 \%$ of the subjects had impaired pulmonary function tests at follow-up.

More than half of the children had been treated with prednisolone, which may have influenced (i.e. accelerated) the recovery. In a previous study on childhood sarcoidosis [14], $81 \%$ of the 21 children were treated with prednisolone. However, their group of patients contained 12 children of black ethnicity, in whom the disease takes a more severe course [1]. Therefore, the two studies are not comparable. 
The present authors conclude that the most frequent initial symptoms/clinical findings in sarcoidosis of Danish children include iridocyclitis, peripheral lymphadenopathy and cutaneous sarcoidosis. In general, childhood sarcoidosis has a favourable prognosis, which is similar to the prognosis in adults. Prognosis appears not to be related to the age at onset of disease. The majority of children recover completely within 6 yrs after onset of disease, but a few recover with persisting organ damage. Some develop chronic active disease and impairment of pulmonary function demanding continuous medical treatment. Approximately $7 \%$ die at a young age due to sarcoidosis-related complications. In general, the presence of erythema nodosum is associated with a good prognosis, and central nervous system sarcoidosis is associated with a poor prognosis.

\section{REFERENCES}

1 Hunninghake GW, Costabel U, Ando M, et al. ATS/ERS/ WASOG statement on sarcoidosis. American Thoracic Society/European Respiratory Society/World Association of Sarcoidosis and other Granulomatous Disorders. Sarcoidosis Vasc Diffuse Lung Dis 1999; 16: 149-173.

2 Byg KE, Milman N, Hansen S. Sarcoidosis in Denmark 1980-1994. A registry-based incidence study comprising 5536 patients. Sarcoidosis Vasc Diffuse Lung Dis 2003; 20: $46-52$.

3 Hoffmann AL, Milman N, Byg KE. Childhood sarcoidosis in Denmark 1979-1994: incidence, clinical features and laboratory results at presentation in 48 children. Acta Paediatr 2004; 93: 30-36.

4 Scadding JG, Mitchell DN. Sarcoidosis. 2nd Edn. London, Chapman and Hall, 1985.

5 Milman N, Selroos O. Pulmonary sarcoidosis in the Nordic countries 1950-1982. Epidemiology and clinical picture. Sarcoidosis 1990; 7: 50-57.
6 Milman N, Selroos O. Pulmonary sarcoidosis in the Nordic countries 1950-1982. II. Course and prognosis. Sarcoidosis 1990; 7: 113-118.

7 Milman N, Andersen CB, Hansen A, et al. Favourable effect of TNF- $\alpha$ inhibitor (infliximab) on Blau syndrome in monozygotic twins with a de novo CARD15 mutation. APMIS 2006; 114: 912-919.

8 Milman N, Hoffmann AL, Byg KE. Sarcoidosis in children. Epidemiology in Danes, clinical features, treatment and prognosis. Acta Pædiatr 1998; 87: 871-878.

9 Shetty AK, Gedalia A. Sarcoidosis in children. Curr Probl Pediatr 2000; 30: 149-176.

10 Kendig EL Jr, Niitu Y. Sarcoidosis in Japanese and American children. Chest 1980; 77: 514-516.

11 James DG, Neville E, Siltzbach LE. A worldwide review of sarcoidosis. Ann NY Acad Sci 1976; 278: 321-334.

12 Pattishall EN, Strope GL, Spinola SM, Denny FW. Childhood sarcoidosis. J Pediatr 1986; 108: 169-177.

13 Kendig EL Jr. The clinical picture of sarcoidosis in children. Pediatrics 1974; 54: 289-292.

14 Baculard A, Blanc N, Boulé M, et al. Pulmonary sarcoidosis in children: a follow-up study. Eur Respir J 2001; 17: 628-635.

15 Hetherington S. Sarcoidosis in young children. Am J Dis Child 1982; 136: 13-15.

16 Pattishall EN, Kendig EL Jr. Sarcoidosis in children. Pediatr Pulmonol 1996; 22: 195-203.

17 Hoffmann AL, Milman N, Nielsen HE, Thode J. Childhood sarcoidosis presenting with hypercalcaemic crisis. Sarcoidosis 1994; 11: 141-143.

18 Nocton JJ, Stork JE, Jacobs G, Newman AJ. Sarcoidosis associated with nephrocalcinosis in young children. J Pediatr 1992; 121: 937-940.

19 Ziegenhagen MW, Rothe ME, Schlaak M, MüllerQuernheim J. Bronchoalveolar and serological parameters reflecting the severity of sarcoidosis. Eur Respir J 2003; 21: 407-413. 